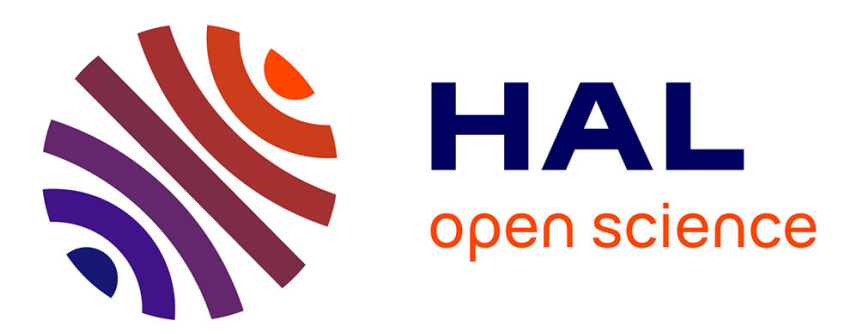

\title{
A Simple Proof of Convergence for an Approximation Scheme for Computing Motions by Mean Curvature
} Guy Barles, Christine Georgelin

\section{To cite this version:}

Guy Barles, Christine Georgelin. A Simple Proof of Convergence for an Approximation Scheme for Computing Motions by Mean Curvature. SIAM Journal on Numerical Analysis, 1995, 32 (2), pp.484500. hal-02315330

\author{
HAL Id: hal-02315330 \\ https://hal.science/hal-02315330
}

Submitted on 14 Oct 2019

HAL is a multi-disciplinary open access archive for the deposit and dissemination of scientific research documents, whether they are published or not. The documents may come from teaching and research institutions in France or abroad, or from public or private research centers.
L'archive ouverte pluridisciplinaire HAL, est destinée au dépôt et à la diffusion de documents scientifiques de niveau recherche, publiés ou non, émanant des établissements d'enseignement et de recherche français ou étrangers, des laboratoires publics ou privés. 


\title{
A SIMPLE PROOF OF CONVERGENCE FOR AN APPROXIMATION SCHEME FOR COMPUTING MOTIONS BY MEAN CURVATURE*
}

\author{
GUY BARLES ${ }^{\dagger}$ AND CHRISTINE GEORGELIN ${ }^{\dagger}$
}

\begin{abstract}
We prove the convergence of an approximation scheme recently proposed by Bence, Merriman, and Osher for computing motions of hypersurfaces by mean curvature. Our proof is based on viscosity solutions methods.
\end{abstract}

Key words. motion by mean curvature, approximation scheme, viscosity solutions

AMS subject classifications. 65N12, 35K65, 53C21, 76T05

1. Introduction. Recently Bence, Merriman, and Osher [4] proposed a very simple (and therefore very tricky) approximation scheme for computing motions of hypersurfaces by mean curvature. The aim of this work is to rigorously show that the discrete motion computed by this scheme actually converges to the continuous motion by mean curvature in some suitable sense.

We first describe the Bence, Merriman, and Osher scheme. Let $\Omega_{0}$ be a smooth bounded domain in $\mathbb{R}^{N}$; one wants to approximate the motion of $\Gamma_{0}=\partial \Omega_{0}$ with a normal velocity equal to its mean curvature. We denote by $h>0$ the size of the time step and we compute for $n \in \mathbb{N}$ functions $u_{h}(., n h)$ defined in $\mathbb{R}^{N}$ in the following way.

- Initialize the process by setting

$$
u_{h}(x, 0)=1_{\Omega_{0}}-1_{\Omega_{0}^{c}} \quad \text { in } \mathbb{R}^{N},
$$

- Define $u_{h}(., n h)$ by induction through the formula

$$
u_{h}(x,(n+1) h)=\operatorname{sign}\left(S(h) u_{h}(., n h)(x)\right),
$$

where the sign function is defined by $\operatorname{sign}(t)=1$ if $t>0,-1$ otherwise and where $S($.) stands for the semigroup associated with the heat equation.

In other words, at each time step, solve the heat equation

$$
\frac{\partial w}{\partial t}-\Delta w=0 \quad \text { in } \mathbb{R}^{N} \times(0, h)
$$

together with the initial data

$$
w(x, 0)=u_{h}(x, n h) \quad \text { in } \mathbb{R}^{N} .
$$

Then define $u_{h}(.,(n+1) h)$ by

$$
u_{h}(x,(n+1) h)= \begin{cases}1 & \text { if } w(x, h)>0 \\ -1 & \text { otherwise. }\end{cases}
$$

This algorithm generates functions $u_{h}(., n h)$ for $n \in \mathbb{N}$ and open sets $\Omega_{n h}^{h}$ defined by

$$
u_{h}(x, n h)=1_{\Omega_{n h}^{h}}-1_{\left(\Omega_{n h}^{h}\right)^{c}} \quad \text { in } \mathbb{R}^{N} .
$$

* Received by the editors December 31, 1992; accepted for publication (in revised form) September $27,1993$.

† Faculté des Sciences et Techniques, Université de Tours, Parc de Grandmont, 37200 Tours, France (barles@univ-tours.fr, georgelin@univ-tours.fr). 
We prove here that, when $h \rightarrow 0$, the discrete motion $\Gamma_{0} \rightarrow \Gamma_{n h}^{h}=\partial \Omega_{n h}^{h}$ converges in a suitable sense to the motion $\Gamma_{0} \rightarrow \Gamma_{t}$, which is the motion with a normal velocity equal to mean curvature.

To be more specific, we first briefly recall a few facts on the recent approach to the problem of motions of fronts. In a series of articles, Evans and Spruck [10] (see also Chen Giga, and Goto [5]) give a rigorous justification of numerical ideas introduced by Osher and Sethian [13] for defining and studying a weak notion of motions of fronts by mean curvature (and, in [5], by other types of geometrical law of propagations). This is the so-called level set approach, which can be described in the following way. Using the notion of viscosity solution of Crandall and Lions [8], it is possible to give a suitable sense to the equation

$$
\frac{\partial u}{\partial t}-\Delta u+\frac{\left(D^{2} u D u \mid D u\right)}{|D u|^{2}}=0 \quad \text { in } \mathbb{R}^{N} \times(0,+\infty),
$$

and to prove that the associated Cauchy problem is well posed in the space of uniformly continuous functions. We refer the reader who is not familiar with viscosity solutions to the "user's guide" of Crandall, Ishii, and Lions [7].

We now denote by $u$ the unique solution of (2) associated with the initial data

$$
u(x, 0)=d\left(x, \Gamma_{0}\right) \quad \text { in } \mathbb{R}^{N},
$$

where $\Gamma_{0}=\partial \Omega_{0}$ and $d$ is the signed distance to $\Gamma_{0}$ which is positive inside $\Omega_{0}$ and negative outside.

The basic result, in this framework, is that the sets $\Omega_{t}=\{u(., t)>0\}$ and $\Gamma_{t}=\{u(., t)=0\}$ do not really depend on the choice of the initial data provided it assumes the same strict signs as $d\left(x, \Gamma_{0}\right)$ in $\mathbb{R}^{N}$. Therefore the evolution of the level set $\Gamma_{0}=\{u(., 0)=0\} \rightarrow \Gamma_{t}=\{u(., t)=0\}$ is purely geometrical since it depends only on $\Gamma_{0}$ and on the sign of the initial data, which make clear the expressions "inside $\Gamma_{0}$ " and "outside $\Gamma_{0} . "$ Moreover one can easily check that, in the case of smooth solutions (typically $u_{0}$ smooth with a smooth 0-level set and for small time) the motion of $\Gamma_{t}$ is indeed a "strong" motion by mean curvature.

However, this approach faces a difficulty due to the fact that $\Gamma_{t}$ may become "fat"; this is the so-called nonempty interior difficulty. This difficulty - but not only this one-will cross our problem in the following way, explained by a result from Barles, Soner, and Souganidis [2]

THEOREM 1.1 ([2]). There exists a unique solution of (2) with initial data $1_{\Omega_{0}}$ $1_{\Omega_{0}^{c}}$ iff $\bigcup_{t>0} \Gamma_{t} \times\{t\}=\partial\{(x, t) ; u(x, t)>0\}=\partial\{(x, t) ; u(x, t)<0\}$.

This result will play a crucial role in our convergence result. Indeed, the problem with initial data $1_{\Omega_{0}}-1_{\Omega_{0}^{c}}$ turns out to be the problem approximated by the Bence, Merriman, and Osher scheme. Since Theorem 1.1 means that this problem is not well posed if the empty interior difficulty occurs, we will get the full convergence only when it doesn't occur. In fact, the condition appearing in Theorem 1.1 is stronger than saying that the set $\bigcup_{t>0} \Gamma_{t} \times\{t\}$ is of empty interior: it rules out configurations where some part of the set $\{u=0\}$ remains included in the interior of the set $\{u \geq 0\}$ or the set $\{u \leq 0\}$ for some time. On the contrary, if this condition holds, this means that the position of the front is known as soon as we know which points are inside the moving front, i.e., the set $\{u>0\}$, or which points are outside the moving front, i.e., the set $\{u<0\}$. A general discussion of this condition and the nonempty interior difficulty is given in [2] and several results are given that ensure that this condition holds for motion by mean curvature and also for different types of motions. 
To state our result, we use the classical notation in the theory of viscosity solutions

$$
\liminf u_{*} u_{h}(x, t)=\liminf _{\substack{y \rightarrow x \\ n h \rightarrow t}} u_{h}(y, n h)
$$

and

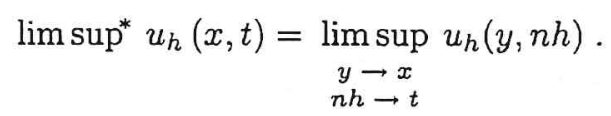

Our result is given in the following theorem.

THEOREM 1.2. Let $\Omega_{t}$ and $\Gamma_{t}$ be defined as above. Then, for all $t \geq 0$

$$
\begin{gathered}
\liminf u_{h}(x, t)=1 \quad \text { in } \Omega_{t}, \\
\limsup u_{h}(x, t)=-1 \quad \text { in }\left(\Omega_{t} \cup \Gamma_{t}\right)^{c} .
\end{gathered}
$$

Theorem 1.2 shows that the scheme gives the correct answer by putting 1 in the region inside the front $(\{u>0\})$ and -1 in the region outside the front $(\{u<0\})$. Conversely, a natural question is whether the regions where $u_{h}$ goes to 1 and -1 are exactly the regions inside and outside the moving front, respectively. In general, the answer is no as it can be seen easily if the front develops an interior. In fact, the answer is yes if and only if the condition of Theorem 1.1 holds. In this case, we have the following more precise corollary, which explains in which sense the discrete front converges to the continuous one. To state it, we denote by $F$ the set $\bigcup_{t>0} \Gamma_{t} \times\{t\}$ and by $F^{h}$ the set $\bigcup_{n \in \mathbb{N}} \Gamma_{n h}^{h} \times\{n h\}$.

Corollary 1.3. If $\bigcup_{t>0} \Gamma_{t} \times\{t\}=\partial\{(x, t) ; u(x, t)>0\}=\partial\{(x, t) ; u(x, t)<$ $0\}$, then $F^{h}$ converges to $F$ in the sense of the Hausdorff distance.

The proof of Theorem 1.2 relies on the general approach for proving convergence of numerical schemes by viscosity solutions methods presented in Barles and Souganidis [3]. The surprising fact here is that the difficulty comes from the consistency requirement on the scheme and the main step in our proof will be to prove this consistency.

After our work was completed, we learned that L. C. Evans [9] obtained the same result using a nonlinear semigroup approach. Our difficulty with the consistency requirement appears in his work for the determination of the infinitesimal generator of the limiting semigroup. Computations related to ours have also been made by $P$. Mascarenhas [12], who checked that for smooth initial fronts the speed is correct, which is again related to our consistency difficulty.

2. On the definition of viscosity solutions for the mean curvature equation. The aim of this section is to give definition equivalent to the classical one since we need it to prove the consistency of the scheme. Definition 2.1 recalls the classical one.

Definition 2.1. A locally bounded upper semicontinuous (usc) (respectively, lower semicontinuous (lsc)) function $u$ (respectively, $v$ ) is a viscosity subsolution (respectively, supersolution) of (2) iff $\forall \phi \in C^{2}\left(\mathbb{R}^{N} \times(0,+\infty)\right)$, if $(x, t) \in \mathbb{R}^{N} \times(0,+\infty)$ is a local maximum point of $u-\phi$, one has

$$
\frac{\partial \phi}{\partial t}(x, t)-\left(\Delta \phi-\frac{\left(D^{2} \phi D \phi \mid D \phi\right)}{|D \phi|^{2}}\right)(x, t) \leq 0 \quad \text { if } D \phi(x, t) \neq 0,
$$


or

$$
\frac{\partial \phi}{\partial t}(x, t)-\Delta \phi(x, t)+\lambda_{\min }\left(D^{2} \phi(x, t)\right) \leq 0 \quad \text { if } D \phi(x, t)=0,
$$

where $\lambda_{\min }\left(D^{2} \phi(x, t)\right)$ is the least eigenvalue of $D^{2} \phi(x, t)$. (Respectively, $\forall \phi \in C^{2}\left(\mathbb{R}^{N} \times\right.$ $(0,+\infty))$, if $(x, t) \in \mathbb{R}^{N} \times(0,+\infty)$ is a local minimum point of $v-\phi$, one has

$$
\frac{\partial \phi}{\partial t}(x, t)-\left(\Delta \phi-\frac{\left(D^{2} \phi D \phi \mid D \phi\right)}{|D \phi|^{2}}\right)(x, t) \geq 0 \quad \text { if } D \phi(x, t) \neq 0,
$$

or

$$
\frac{\partial \phi}{\partial t}(x, t)-\Delta \phi(x, t)+\lambda_{\max }\left(D^{2} \phi(x, t)\right) \geq 0 \quad \text { if } D \phi(x, t)=0,
$$

where $\lambda_{\max }\left(D^{2} \phi(x, t)\right)$ is the principal eigenvalue of $\left.D^{2} \phi(x, t).\right)$

This definition comes from the general definition of viscosity solution for discontinuous Hamiltonians first given by Ishii [11] (See also the "user's guide" [7]). Here we have to treat the discontinuity which appears in the equation for $D \phi(x, t)=0$. We recall that, in this definition, we may replace "local" by "global" or "local" by "local strict" and "global" by "global strict" without changing the notion. We will often use this capability.

We need an equivalent definition which eliminates, at least partially, the difficulty related to the fact that $D \phi(x, t)$ may be equal to 0 .

Proposition 2.2. A locally bounded usc (respectively, lsc) function $u$ (respectively, $v$ ) is a viscosity subsolution (respectively, supersolution) of (2) iff it satisfies (4) and

$$
\frac{\partial \phi}{\partial t}(x, t) \leq 0 \quad \text { if } \quad D \phi(x, t)=0 \quad \text { and } \quad D^{2} \phi(x, t)=0,
$$

respectively, (6) and

$$
\frac{\partial \phi}{\partial t}(x, t) \geq 0 \quad \text { if } \quad D \phi(x, t)=0 \quad \text { and } \quad D^{2} \phi(x, t)=0 .
$$

The result of the proposition implies that no property has to be satisfied when $D \phi(x, t)=0$ and $D^{2} \phi(x, t) \neq 0$; this will simplify our checking of the consistency requirement.

Proof. We treat only the subsolution case since the other one is completely analogous. Let $u$ be a locally bounded function which satisfies (4) and $\left(5^{\prime}\right)$. Let $\phi$ be a $C^{2}$-function on $\mathbb{R}^{N} \times(0,+\infty)$ and $(x, t)$ be a strict local maximum point of $u-\phi$. The only difficulty is when $D \phi(x, t)=0$ and $D^{2} \phi(x, t) \neq 0$ since no property is a priori supposed to be satisfied by $u$ in this case.

In this case, we consider the function

$$
\psi_{\varepsilon}(x, y, t)=u(x, t)-\frac{|x-y|^{4}}{\varepsilon}-\phi(y, t)
$$

where $\varepsilon$ is a parameter devoted to go to 0 . Since $(x, t)$ is a strict maximum point of $u-\phi$, one proves easily that there is a sequence $\left(x_{\varepsilon}, y_{\varepsilon}, t_{\varepsilon}\right)$ of local maximum points of $\psi_{\varepsilon}$ converging to $(x, x, t)$. We have in the $y$ variable the classical properties of a local maximum point

$$
D \phi\left(y_{\varepsilon}, t_{\varepsilon}\right)=\frac{4\left(x_{\varepsilon}-y_{\varepsilon}\right)\left|x_{\varepsilon}-y_{\varepsilon}\right|^{2}}{\varepsilon}
$$


and

$$
D^{2} \phi\left(y_{\varepsilon}, t_{\varepsilon}\right) \geq-\frac{4\left|x_{\varepsilon}-y_{\varepsilon}\right|^{2}}{\varepsilon} I d-\frac{8\left(x_{\varepsilon}-y_{\varepsilon}\right) \otimes\left(x_{\varepsilon}-y_{\varepsilon}\right)}{\varepsilon} .
$$

Two cases may occur.

1. $D \phi\left(y_{\varepsilon}, t_{\varepsilon}\right)=0$. This implies $x_{\varepsilon}=y_{\varepsilon}$. We fix $y=y_{\varepsilon}$ and we argue in the $x$-variable: using that $u$ satisfies the property $\left(5^{\prime}\right)$ we get

$$
\frac{\partial \phi}{\partial t}\left(y_{\varepsilon}, t_{\varepsilon}\right) \leq 0,
$$

but since $D^{2} \phi\left(y_{\varepsilon}, t_{\varepsilon}\right) \geq 0$, this implies

$$
\frac{\partial \phi}{\partial t}\left(y_{\varepsilon}, t_{\varepsilon}\right)-\Delta \phi\left(y_{\varepsilon}, t_{\varepsilon}\right)+\lambda_{\min }\left(D^{2} \phi\left(y_{\varepsilon}, t_{\varepsilon}\right)\right) \leq 0,
$$

and we complete the proof by letting $\varepsilon$ go to 0 .

2. $D \phi\left(y_{\varepsilon}, t_{\varepsilon}\right) \neq 0$. We remark that $\left(x_{\varepsilon}, t_{\varepsilon}\right)$ is a maximum point of

$$
(x, t) \mapsto u(x, t)-\frac{\left|x_{\varepsilon}-y_{\varepsilon}\right|^{4}}{\varepsilon}-\phi\left(x-\left(x_{\varepsilon}-y_{\varepsilon}\right), t\right),
$$

and using (4), we obtain

$$
\frac{\partial \phi}{\partial t}\left(y_{\varepsilon}, t_{\varepsilon}\right)-\left(\Delta \phi+\frac{\left(D^{2} \phi D \phi \mid D \phi\right)}{|D \phi|^{2}}\right)\left(y_{\varepsilon}, t_{\varepsilon}\right) \leq 0 .
$$

We conclude by letting $\varepsilon$ go to 0 , remarking that

$$
\liminf _{\varepsilon} \frac{\left(D^{2} \phi D \phi \mid D \phi\right)}{|D \phi|^{2}}\left(y_{\varepsilon}, t_{\varepsilon}\right) \geq \lambda_{\min }\left(D^{2} \phi(y, t)\right) .
$$

The proof is complete.

3. The convergence proof. We borrow here arguments from [3] to be as selfcontained as possible.

The main step consists in proving the following proposition.

Proposition 3.1. The functions limsup* $u_{h}$ and $\liminf _{*} u_{h}$ are, respectively, viscosity sub- and supersolutions of (2).

We first complete the proof of Theorem 1.2 assuming that the result of Proposition 3.1 holds. The basic tool is the "uniqueness" result of [2]. Indeed, if $u$ is defined as in the introduction and if we denote by sign* and by $\operatorname{sign}_{*}$ the usc envelope and the lsc envelope, respectively, of the sign function in $\mathbb{R}$, it is proved in [2] that

$$
\operatorname{sign}^{*}(u(x, t))= \begin{cases}1 & \text { if } u(x, t) \geq 0 \\ -1 & \text { otherwise }\end{cases}
$$

and

$$
\operatorname{sign}_{*}(u(x, t))= \begin{cases}1 & \text { if } u(x, t)>0 \\ -1 & \text { otherwise }\end{cases}
$$

are the maximal subsolution and the minimal supersolution, respectively, of (2) with initial data $1_{\Omega_{0}}-1_{\Omega_{0}^{c}}$. Therefore any subsolution $v$ and any supersolution $w$ of (2) with initial data $1_{\Omega_{0}}-1_{\Omega_{0}^{c}}$ satisfy

$$
v(x, t) \leq \operatorname{sign}^{*}(u(x, t)) \quad \text { in } \mathbb{R}^{N} \times(0,+\infty),
$$


and

$$
w(x, t) \geq \operatorname{sign}_{*}(u(x, t)) \quad \text { in } \mathbb{R}^{N} \times(0,+\infty) .
$$

We apply these inequalities to $\lim \sup ^{*} u_{h}$ and $\liminf { }_{*} u_{h}$, respectively:

$$
\lim \sup ^{*} u_{h}(x, t) \leq \operatorname{sign}^{*}(u(x, t)) \quad \text { in } \mathbb{R}^{N} \times(0,+\infty)
$$

and

$$
\liminf { }_{*} u_{h}(x, t) \geq \operatorname{sign}_{*}(u(x, t)) \quad \text { in } \mathbb{R}^{N} \times(0,+\infty) .
$$

Since $u_{h}$ takes values in $\{-1,1\}$, inequality (9) implies that $\liminf _{*} u_{h}=1$ in $\Omega_{t}=$ $\{u>0\}$ while inequality (8) implies that limsup* $u_{h}=-1$ in $\{u<0\}$. The proof of Theorem 1.2 is complete.

Notice that another consequence of (8) and (9) is that, where $\lim \sup ^{*} u_{h}(x, t)=1$, $\operatorname{sign}^{*}(u(x, t))=1$ or equivalently $u(x, t) \geq 0$ and, in the same way, $\operatorname{sign}_{*}(u(x, t))=-1$ or $u(x, t) \leq 0$ where $\liminf _{*} u_{h}(x, t)=-1$. If the condition of Theorem 1.1 holds, the set $\bigcup_{t>0} \Gamma_{t} \times\{t\}$ just appears as the boundary of the sets where $u_{h}$ goes respectively to 1 and -1 and our result is really complete in this case. Otherwise, we lose some information: if $\bigcup_{t>0} \Gamma_{t} \times\{t\}$ has a nonempty interior, we do not know the asymptotic behavior of $u_{h}$ on it. If there are parts of the moving front inside the interior of the sets $\{u \geq 0\}$ or $\{u \leq 0\}$, they can be dropped out by the scheme.

Now we turn to the proof of Proposition 3.1. We detail only the case of lim sup* $u_{h}$, since the other one is completely analogous. Curiously the difficulty will come from the consistency requirement, which is usually the easiest property to be checked.

We set $\bar{u}=\lim \sup ^{*} u_{h}$. Let $\phi$ be a smooth test function and let $(x, t) \in \mathbb{R}^{N} \times$ $(0,+\infty)$ be a strict global maximum point of $\bar{u}-\phi$. Since the notion of viscosity solution is local, we may assume without loss of generality that

$$
\liminf _{|x|+t \rightarrow+\infty} \phi(x, t)=+\infty .
$$

This property will eliminate any difficulty coming from the unboundedness of the domain.

If $\bar{u}(x, t)=-1$, since $\bar{u}$ is usc and takes values in $\{-1,1\}$, then $\bar{u}=-1$ in a neighborhood of $(x, t)$ and thus

$$
D \phi(x, t)=0, \quad D^{2} \phi(x, t) \geq 0, \quad \frac{\partial \phi}{\partial t}(x, t)=0,
$$

and therefore we have

$$
\frac{\partial \phi}{\partial t}(x, t)-\Delta \phi(x, t)+\lambda_{\min }\left(D^{2} \phi(x, t) \leq 0 .\right.
$$

The conclusion is obtained in the same way if $(x, t)$ is in the interior of the set $\{\bar{u}=1\}$. So we may assume that $(x, t)$ is at the boundary of this set.

We first notice that limsup* $u_{h}=\lim \sup ^{*} u_{h}^{*}$ and therefore we can work as well with $u_{h}^{*}$. By Lemma A.3 in the Appendix in Barles and Perthame [1] and because of (10), there is a subsequence $\left(x_{h}, n_{h} h\right)$ converging to $(x, t)$ such that

$$
u_{h}^{*}\left(x_{h}, n_{h} h\right)-\phi\left(x_{h}, n_{h} h\right)=\max _{\mathbb{R}^{N} \times \mathbb{N}}\left(u_{h}^{*}-\phi\right),
$$


and

$$
u_{h}^{*}\left(x_{h}, n_{h} h\right) \rightarrow 1 .
$$

We use here the fact that $u_{h}^{*}$ is usc and this is the reason for considering it.

Since $u_{h}$ takes its values in $\{-1,1\}$, the convergence of $u_{h}^{*}\left(x_{h}, n_{h} h\right)$ to 1 implies that $u_{h}^{*}\left(x_{h}, n_{h} h\right)=1$ for $h$ small enough. Moreover, for such $h$, the fact that $\left(x_{h}, n_{h} h\right)$ is a maximum point reads

$$
u_{h}^{*}(x, n h) \leq 1-\phi\left(x_{h}, n_{h} h\right)+\phi(x, n h),
$$

for all $x \in \mathbb{R}^{N}$ and $n \in \mathbb{N}$.

The consequence is the following: if $u_{h}^{*}(x, n h)=1$, necessarily $\phi(x, n h)-\phi\left(x_{h}, n_{h} h\right)$ $\geq 0$ and finally

$$
u_{h}^{*}(x, n h) \leq \operatorname{sign}^{*}\left(\phi(x, n h)-\phi\left(x_{h}, n_{h} h\right)\right) .
$$

If $u_{h}^{*}(x, n h)=-1$, the same inequality is true. Moreover we notice that, since

$$
u_{h}\left(x, n_{h} h\right)=\operatorname{sign}^{*}\left(S(h) u_{h}\left(.,\left(n_{h}-1\right) h\right)\right)(x) \quad \text { in } \mathbb{R}^{N},
$$

we clearly have

$$
u_{h}\left(x, n_{h} h\right) \leq \operatorname{sign}^{*}\left(S(h) u_{h}^{*}\left(.,\left(n_{h}-1\right) h\right)\right)(x) \quad \text { in } \mathbb{R}^{N},
$$

and finally, since the right-hand side is usc,

$$
u_{h}^{*}\left(x, n_{h} h\right) \leq \operatorname{sign}^{*}\left(S(h) u_{h}^{*}\left(.,\left(n_{h}-1\right) h\right)\right)(x) \quad \text { in } \mathbb{R}^{N} .
$$

Therefore, using this property for $x=x_{h}$ and according to the monotonicity of $S(h)$ and the sign function, we have

$$
\begin{aligned}
1=u_{h}^{*}\left(x_{h}, n_{h} h\right) & \leq \operatorname{sign}^{*}\left(S(h) u_{h}^{*}\left(.,\left(n_{h}-1\right) h\right)\right)\left(x_{h}\right) \\
& \leq \operatorname{sign}^{*}\left(S(h)\left[\operatorname{sign}^{*}\left(\phi\left(.,\left(n_{h}-1\right) h\right)-\phi\left(x_{h}, n_{h} h\right)\right)\right]\left(x_{h}\right)\right),
\end{aligned}
$$

and this is equivalent to

$$
\left.S(h)\left[\operatorname{sign}^{*}\left(\phi\left(.,\left(n_{h}-1\right) h\right)\right)-\phi\left(x_{h}, n_{h} h\right)\right)\right]\left(x_{h}\right) \geq 0 .
$$

The remainder of the proof consists of proving that this last inequality implies

$$
\frac{\partial \phi}{\partial t}(x, t)-\left(\Delta \phi+\frac{\left(D^{2} \phi D \phi \mid D \phi\right)}{|D \phi|^{2}}\right)(x, t) \leq 0 \quad \text { if } D \phi(x, t) \neq 0
$$

or

$$
\frac{\partial \phi}{\partial t}(x, t) \leq 0 \quad \text { if } D \phi(x, t)=0 \text { and } D^{2} \phi(x, t)=0,
$$

according to the equivalent definition we gave in Proposition 2.2 .

Remark. By the construction of the scheme, $u_{h}$ is lsc and therefore the arguments for the supersolution are simpler (construction of minimum point, etc.). 
4. Consistency of the scheme. To simplify the proof of (12) and (13), we are going to replace the sign function by the function $(\operatorname{sign}(x)+1) / 2$. Essentially -1 will be replaced by 0,1 remains 1 , and the central role of 0 is now played by $\frac{1}{2}$. We denote below by $C^{2,1}\left(\mathbb{R}^{N} \times(0,+\infty)\right)$ the space of $C^{2}$ functions in $x$ and $t$ with Lipschitz continuous second derivatives.

We prove the following proposition.

PROPOSITION 4.1. If $\left(\phi_{h}\right)_{h}$ is a sequence of smooth functions bounded in $C^{2,1}$ and converging locally in $C^{2,1}$ to a function $\phi$ and $\left(x_{h}, t_{h}\right)$ is a sequence of points converging to $(x, t) \in \mathbb{R}^{N} \times(0,+\infty)$ s.t. $\phi_{h}\left(x_{h}, t_{h}\right)=0$ then, if $D \phi(x, t) \neq 0$,

$$
\begin{aligned}
\liminf _{h} \frac{1}{h^{1 / 2}}( & \left.\frac{1}{2}-\frac{1}{(4 \pi h)^{N / 2}} \int_{\left\{\phi_{h}\left(., t_{h}-h\right) \geq 0\right\}} \exp \left(-\frac{\left|x_{h}-y\right|^{2}}{4 h}\right) d y\right) \\
& \geq \frac{1}{2 \sqrt{\pi}|D \phi(x, t)|}\left(\frac{\partial \phi}{\partial t}-\Delta \phi+\frac{\left(D^{2} \phi D \dot{\phi} \mid D \phi\right)}{|D \phi|^{2}}\right)(x, t) .
\end{aligned}
$$

Moreover, if $D \phi(x, t)=0$ and $D^{2} \phi(x, t)=0$ and if the inequality

$$
\frac{1}{2}-\frac{1}{(4 \pi h)^{N / 2}} \int_{\left\{\phi_{h}\left(., t_{h}-h\right) \geq 0\right\}} \exp \left(-\frac{\left|x_{h}-y\right|^{2}}{4 h}\right) d y \leq 0
$$

holds for a sequence of $h$ converging to 0 , then

$$
\frac{\partial \phi}{\partial t}(x, t) \leq 0 .
$$

We first complete the proof of Proposition 3.1 using the result of Proposition 4.1. We first notice

$$
\begin{aligned}
& S(h)\left(\frac{1+\operatorname{sign}^{*}}{2}\right)\left(\phi\left(.,\left(n_{h}-1\right) h\right)-\phi\left(x_{h}, n_{h} h\right)\right) \\
& \quad=\frac{1}{(4 \pi h)^{N / 2}} \int_{\left\{\phi\left(.,\left(n_{h}-1\right) h\right)-\phi\left(x_{h}, n_{h} h\right) \geq 0\right\}} \exp \left(-\frac{\left|x_{h}-y\right|^{2}}{4 h}\right) d y .
\end{aligned}
$$

And therefore (11) reads

$$
\frac{1}{(4 \pi h)^{N / 2}} \int_{\left\{\phi\left(.,\left(n_{h}-1\right) h\right)-\phi\left(x_{h}, n_{h} h\right) \geq 0\right\}} \exp \left(-\frac{\left|x_{h}-y\right|^{2}}{4 h}\right) d y \geq \frac{1}{2},
$$

or equivalently

$$
\frac{1}{2}-\frac{1}{(4 \pi h)^{N / 2}} \int_{\left\{\phi\left(.,\left(n_{h}-1\right) h\right)-\phi\left(x_{h}, n_{h} h\right) \geq 0\right\}} \exp \left(-\frac{\left|x_{h}-y\right|^{2}}{4 h}\right) d y \leq 0 .
$$

Now we apply Proposition 4.1 with $\phi_{h}(x, t)=\phi(x, t)-\phi\left(x_{h}, n_{h} h\right), t_{h}=n_{h} h$ and $x_{h}$. If $D \phi(x, t) \neq 0$ then

$$
\begin{aligned}
0 \geq \liminf _{h} \frac{1}{h^{1 / 2}}\left(\frac{1}{2}\right. & \left.-\frac{1}{(4 \pi h)^{N / 2}} \int_{\left\{\phi_{h}\left(., t_{h}-h\right) \geq 0\right\}} \exp \left(-\frac{\left|x_{h}-y\right|^{2}}{4 h}\right) d y\right) \\
& \geq \frac{1}{2 \sqrt{\pi}|D \phi(x, t)|}\left(\frac{\partial \phi}{\partial t}-\Delta \phi+\frac{\left(D^{2} \phi D \phi \mid D \phi\right)}{|D \phi|^{2}}\right)(x, t) .
\end{aligned}
$$


And finally

$$
\frac{\partial \phi}{\partial t}(x, t)-\left(\Delta \phi-\frac{\left(D^{2} \phi D \phi \mid D \phi\right)}{|D \phi|^{2}}\right)(x, t) \leq 0
$$

The conclusion is even easier in the case when $D \phi(x, t)=0$ and $D^{2} \phi(x, t)=0$.

Proof of Proposition 4.1. In the integral, we make the change

$$
z=\frac{y-x_{h}}{2 \sqrt{h}}
$$

and we set $\eta=\sqrt{h}$. The integral becomes

$$
\frac{1}{(\pi)^{N / 2}} \int_{\left\{\phi_{h}\left(x_{h}+2 \eta z, t_{h}-\eta^{2}\right)>0\right\}} \exp \left(-|z|^{2}\right) d z .
$$

Then we examine the function $\phi_{h}\left(x_{h}+2 \eta z, t_{h}-\eta^{2}\right)$. Since $\phi_{h}\left(x_{h}, t_{h}\right)=0$ and $\phi_{h}$ is bounded in $C^{2,1}$, we have

$$
\phi_{h}\left(x_{h}+2 \eta z, t_{h}-\eta^{2}\right)=2 \eta\left(p_{h}, z\right)+2 \eta^{2}\left(\left(A_{h} z, z\right)-a_{h}+O\left(\eta|z|+\eta^{2}\right)\left(|z|^{2}+\eta^{2}\right)\right),
$$

where $p_{h}, A_{h}, a_{h}$ are identified as

$$
\begin{aligned}
& p_{h}=D \phi_{h}\left(x_{h}, t_{h}\right), \\
& A_{h}=D^{2} \phi_{h}\left(x_{h}, t_{h}\right),
\end{aligned}
$$

and

$$
a_{h}=\frac{1}{2} \frac{\partial \phi_{h}}{\partial t}\left(x_{h}, t_{h}\right)
$$

We use now the change $\tilde{z}=O_{h} z$ where $O_{h}$ is a matrix of rotation chosen in such a way to have $O_{h} p_{h}=\beta_{h}(1,0, \ldots, 0)$ where $\beta_{h}=\left|p_{h}\right|$. Moreover we may assume without loss of generality that $O_{h}$ converges to a matrix of rotation $O$. In the new variables, the set $\left\{\phi_{h}\left(x_{h}+2 \eta z, t_{h}-\eta^{2}\right) \geq 0\right\}$ becomes the set

$$
\left\{2 \eta \beta_{h} \tilde{z}_{1}+2 \eta^{2}\left(\left(\tilde{A}_{h} \tilde{z}, \tilde{z}\right)-a_{h}+O\left(\eta|\tilde{z}|+\eta^{2}\right)\left(|\tilde{z}|^{2}+\eta^{2}\right)\right) \geq 0\right\}
$$

where $\tilde{A}_{h}=O_{h} A_{h} O_{h}^{-1}$.

From now on, we will denote the new variable by $z$ instead of $\tilde{z}$. Dividing the function that we consider by $2 \eta$, which is a positive number, the above set can again be written as

$$
\left\{\beta_{h} z_{1}+\eta\left(\left(\tilde{A}_{h} z, z\right)-a_{h}+O\left(\eta|z|+\eta^{2}\right)\left(|z|^{2}+\eta^{2}\right)\right) \geq 0\right\} .
$$

We first treat the case when $D \phi(x, t) \neq 0$. Since $\beta_{h}=\left|D \phi_{h}\left(x_{h}, t_{h}\right)\right| \rightarrow|D \phi(x, t)|$ as $h \rightarrow 0, \beta_{h}$ is strictly positive if $h$ is small enough. Therefore, dividing the expression inside by $\beta_{h}$, the set

$$
\left\{\beta_{h} z_{1}+\eta\left(\left(\tilde{A}_{h} z, z\right)-a_{h}+O\left(\eta|z|+\eta^{2}\right)\left(|z|^{2}+\eta^{2}\right)\right) \geq 0\right\}
$$


may again be replaced by

$$
\left\{z_{1}+\frac{\eta}{\beta_{h}}\left(\left(\tilde{A}_{h} z, z\right)-a_{h}+O\left(\eta|z|+\eta^{2}\right)\left(|z|^{2}+\eta^{2}\right)\right) \geq 0\right\}
$$

Moreover, thanks to the local convergence of $\phi_{h}$ to $\phi$ in $C^{2,1}$, one has

$$
\tilde{A}_{h} \rightarrow \tilde{A},
$$

and

$$
a_{h} \rightarrow a
$$

as $h \rightarrow 0$, where $\tilde{A}=O D^{2} \phi(x, t) O^{-1}$ and $a=\frac{1}{2} \frac{\partial \phi}{\partial t}(x, t)$. So the set we consider can finally be written as

$$
\left\{z_{1}+\frac{\eta}{\beta_{h}}\left((\tilde{A} z, z)-a+O\left(\eta|z|+\eta^{2}\right)\left(|z|^{2}+\eta^{2}\right)+o(1)\left(|z|^{2}+1\right)\right) \geq 0\right\} .
$$

So within some perturbation terms, we are essentially interested in the behavior for $s$ close to 0 of the function $\varphi$ defined by

$$
\varphi(s)=\frac{1}{(\pi)^{N / 2}} \int_{\left\{z_{1}+s \psi(z) \geq 0\right\}} \exp \left(-|z|^{2}\right) d z
$$

where $\psi(z)=(\tilde{A} z, z)-a, a \in \mathbb{R}$, and $\tilde{A}=\left(\tilde{A}_{i, j}\right)_{i, j}$ is a symmetric matrix. This behavior is described in the following lemma.

Lemma 4.2. One has

$$
\varphi(s)=\frac{1}{2}-\frac{1}{\sqrt{\pi}}\left(a-\frac{1}{2}\left(\operatorname{Tr}(\tilde{A})-\tilde{A}_{1,1}\right)\right) s+o(s) .
$$

We first prove Lemma 4.2. Because of the symmetries one sees easily that $\varphi(0)=$ $\frac{1}{2}$. To get the next term, we are going to prove that $\varphi$ is differentiable at 0 and compute $\varphi^{\prime}(0)$.

The function $\varphi$ can be written as

$$
\varphi(s)=\frac{1}{(\pi)^{N / 2}} \int_{\left\{z_{1}+s \psi(z) \geq 0\right\}} \exp \left(-|z|^{2}\right) d z=\frac{1}{(\pi)^{N / 2}} \int_{\mathbb{R}^{N}} 1_{\left\{z_{1}+s \psi(z) \geq 0\right\}} \exp \left(-|z|^{2}\right) d z .
$$

To prove that $\varphi$ is a $C^{1}$ function of $s$, instead of computing its derivative in the sense of distributions, we approximate $\varphi$ by $\varphi_{\varepsilon}$ defined by

$$
\varphi_{\varepsilon}(s)=\frac{1}{(\pi)^{N / 2}} \int_{\mathbb{R}^{N}} \frac{1}{2}(1+\tanh )\left(\frac{z_{1}+s \psi(z)}{\varepsilon}\right) \exp \left(-|z|^{2}\right) d z .
$$

By standard results, $\varphi_{\varepsilon}$ is a $C^{1}$ function of $s$ and

$$
\varphi_{\varepsilon}^{\prime}(s)=\frac{1}{(\pi)^{N / 2}} \int_{\mathbb{R}^{N}} \frac{1}{2 \varepsilon}\left(1-\tanh ^{2}\right)\left(\frac{z_{1}+s \psi(z)}{\varepsilon}\right) \psi(z) \exp \left(-|z|^{2}\right) d z .
$$

To proceed in the computation, we remark that

$$
\begin{aligned}
\frac{1}{\varepsilon}\left(1-\tanh ^{2}\right)\left(\frac{z_{1}+s \psi(z)}{\varepsilon}\right)= & \frac{\partial}{\partial z_{1}}\left((1+\tanh )\left(\frac{z_{1}+s \psi(z)}{\varepsilon}\right)\right) \\
& -\frac{1}{\varepsilon}\left(1-\tanh ^{2}\right)\left(\frac{z_{1}+s \psi(z)}{\varepsilon}\right) s \frac{\partial \psi}{\partial z_{1}}(z) .
\end{aligned}
$$


Now we use the equality

$$
\varphi_{\varepsilon}(t)-\varphi_{\varepsilon}(0)=\int_{0}^{t} \varphi_{\varepsilon}^{\prime}(s) d s,
$$

which gives

$$
\begin{gathered}
\varphi_{\varepsilon}(t)-\varphi_{\varepsilon}(0)=\frac{1}{(\pi)^{N / 2}} \int_{0}^{t} \int_{\mathbb{R}^{N}} \frac{1}{2} \frac{\partial}{\partial z_{1}}\left((1+\tanh )\left(\frac{z_{1}+s \psi(z)}{\varepsilon}\right)\right) \psi(z) \exp \left(-|z|^{2}\right) d z d s \\
-\frac{1}{(\pi)^{N / 2}} \int_{0}^{t} \int_{\mathbb{R}^{N}} \frac{1}{2 \varepsilon}\left(1-\tanh ^{2}\right)\left(\frac{z_{1}+s \psi(z)}{\varepsilon}\right) s \frac{\partial \psi}{\partial z_{1}}(z) \psi(z) \exp \left(-|z|^{2}\right) d z d s .
\end{gathered}
$$

Then we integrate by parts in the $z_{1}$ variable in the first integral and, after using Fubini's theorem, we integrate by parts in $s$ in the second one to get

$$
\begin{aligned}
\varphi_{\varepsilon}(t)-\varphi_{\varepsilon}(0)= & -\frac{1}{(\pi)^{N / 2}} \int_{0}^{t} \int_{\mathbb{R}^{N}} \frac{1}{2}(1+\tanh )\left(\frac{z_{1}+s \psi(z)}{\varepsilon}\right) \frac{\partial}{\partial z_{1}}\left(\psi(z) \exp \left(-|z|^{2}\right)\right) d z d s \\
& -\frac{1}{(\pi)^{N / 2}} t \int_{\mathbb{R}^{N}} \frac{1}{2}(1+\tanh )\left(\frac{z_{1}+t \psi(z)}{\varepsilon}\right) \frac{\partial \psi}{\partial z_{1}}(z) \exp \left(-|z|^{2}\right) d z \\
& +\frac{1}{(\pi)^{N / 2}} \int_{0}^{t} \int_{\mathbb{R}^{N}} \frac{1}{2}(1+\tanh )\left(\frac{z_{1}+s \psi(z)}{\varepsilon}\right) \frac{\partial \psi}{\partial z_{1}}(z) \exp \left(-|z|^{2}\right) d z d s
\end{aligned}
$$

From the Lebesgue's dominated convergence theorem, we infer that

$$
\begin{aligned}
\varphi(t)-\varphi(0)= & -\frac{1}{(\pi)^{N / 2}} \int_{0}^{t} \int_{\mathbb{R}^{N}} 1_{\left\{z_{1}+s \psi(z) \geq 0\right\}} \frac{\partial}{\partial z_{1}}\left(\psi(z) \exp \left(-|z|^{2}\right)\right) d z d s \\
& -\frac{1}{(\pi)^{N / 2}} t \int_{\mathbb{R}^{N}} 1_{\left\{z_{1}+t \psi(z) \geq 0\right\}} \frac{\partial \psi}{\partial z_{1}}(z) \exp \left(-|z|^{2}\right) d z \\
& +\frac{1}{(\pi)^{N / 2}} \int_{0}^{t} \int_{\mathbb{R}^{N}} 1_{\left\{z_{1}+s \psi(z) \geq 0\right\}} \frac{\partial \psi}{\partial z_{1}}(z) \exp \left(-|z|^{2}\right) d z d s .
\end{aligned}
$$

Since all the integrals over $\mathbb{R}^{N}$ are continuous functions of $s$ or $t$, again because of Lebesgue's theorem, $\varphi^{\prime}(0)$ exists and, by easy computations, we have

$$
\varphi^{\prime}(0)=-\frac{1}{(\pi)^{N / 2}} \int_{\left\{z_{1} \geq 0\right\}} \frac{\partial}{\partial z_{1}}\left(\psi(z) \exp \left(-|z|^{2}\right)\right) d z .
$$

Therefore

$$
\varphi^{\prime}(0)=\frac{1}{(\pi)^{N / 2}} \int_{\mathbb{R}^{N-1}} \psi\left(0, z^{\prime}\right) \exp \left(-\left|z^{\prime}\right|^{2}\right) d z^{\prime},
$$

where $z^{\prime}=\left(z_{2}, \ldots, z_{N}\right)$.

Recall that $\psi\left(0, z^{\prime}\right)=\left(\tilde{A} z^{\prime}, z^{\prime}\right)-a$ where $\tilde{A}=\left(\tilde{A}_{i, j}\right)_{i, j}$ and the equalities

$$
\left.\frac{1}{(\pi)^{N / 2}} \int_{\mathbb{R}^{n-1}} z_{i} z_{j} \exp \left(-\left|z^{\prime}\right|^{2}\right)\right) d z^{\prime}=\frac{1}{2 \sqrt{\pi}} \delta_{i, j}
$$

for $2 \leq i, j \leq n$ and where $\delta_{i, j}=0$ if $i \neq j, 1$ otherwise. Fortunately we can compute explicitly $\varphi^{\prime}(0)$, which is given by

$$
\varphi^{\prime}(0)=-\frac{1}{\sqrt{\pi}}\left(a-\frac{1}{2}\left(\operatorname{Tr}(\tilde{A})-\tilde{A}_{1,1}\right)\right)
$$


The proof of Lemma 4.2 is complete.

Now we come back to the proof of Proposition 4.1. We have to consider the integral

$$
\frac{1}{(\pi)^{N / 2}} \int_{\left\{\Psi_{h}(z) \geq 0\right\}} \exp \left(-|z|^{2}\right) d z=\frac{1}{(\pi)^{N / 2}} \int_{\mathbb{R}^{N}} 1_{\left\{\Psi_{h}(z) \geq 0\right\}} \exp \left(-|z|^{2}\right) d z
$$

where

$$
\Psi_{h}(z)=z_{1}+\frac{\eta}{\beta_{h}}\left((\tilde{A} z, z)-a+O\left(\eta|z|+\eta^{2}\right)\left(|z|^{2}+\eta^{2}\right)+o(1)\left(|z|^{2}+1\right)\right) .
$$

To prove the result, we are going to use a "localization type argument" and then a "monotonicity type argument."

Let us first describe the localization type argument. We set $R=\eta^{-1 / 2}$ and split the integral in two terms:

$$
\begin{aligned}
\frac{1}{(\pi)^{N / 2}} \int_{\mathbb{R}^{N}} 1_{\left\{\Psi_{h}(z) \geq 0\right\}} \exp \left(-|z|^{2}\right) d z= & \frac{1}{(\pi)^{N / 2}} \int_{B_{R}} 1_{\left\{\Psi_{h}(z) \geq 0\right\}} \exp \left(-|z|^{2}\right) d z \\
& +\frac{1}{(\pi)^{N / 2}} \int_{B_{R}^{c}} 1_{\left\{\Psi_{h}(z) \geq 0\right\}} \exp \left(-|z|^{2}\right) d z .
\end{aligned}
$$

The point is that the second integral of the right-hand side is estimated by

$$
\frac{1}{(\pi)^{N / 2}} \int_{B_{R}^{c}} \exp \left(-|z|^{2}\right) d z=\circ(\eta)
$$

Moreover, if $z \in B_{R}$, the $O(\eta|z|)$ term in $\Psi_{h}$ is estimated uniformly in h by an $O(\eta R)$ and therefore is a $\circ(1)$ term.

We now turn to the monotonicity type argument. We consider $\gamma>0$ and examine the function

$$
\Psi(z)=z_{1}+\frac{\eta}{\beta_{h}}(((\tilde{A}+\gamma I d) z, z)-a+\gamma) .
$$

By the above remarks we know that, on $B_{R}$,

$$
\Psi_{h}(z)=z_{1}+\frac{\eta}{\beta_{h}}\left((\tilde{A} z, z)-a+\circ(1)|z|^{2}+\circ(1)\right) .
$$

Hence, for $\eta$ and thus $h$ small enough,

$$
\Psi(z) \geq \Psi_{h}(z) \text { in } B_{R},
$$

and therefore

$$
\frac{1}{(\pi)^{N / 2}} \int_{B_{R}} 1_{\left\{\Psi_{h}(z) \geq 0\right\}} \exp \left(-|z|^{2}\right) d z \leq \frac{1}{(\pi)^{N / 2}} \int_{B_{R}} 1_{\{\Psi(z) \geq 0\}} \exp \left(-|z|^{2}\right) d z .
$$

Finally, using our localization type argument backwards, we obtain

$$
\frac{1}{(\pi)^{N / 2}} \int_{\mathbb{R}^{N}} 1_{\left\{\Psi_{h}(z) \geq 0\right\}} \exp \left(-|z|^{2}\right) d z \leq \frac{1}{(\pi)^{N / 2}} \int_{\mathbb{R}^{N}} 1_{\{\Psi(z) \geq 0\}} \exp \left(-|z|^{2}\right) d z+\circ(\eta) .
$$


To conclude, we apply Lemma 4.2 to the integral of the right-hand side with $s=\frac{\eta}{\beta_{h}}$. We get

$$
\begin{aligned}
& \frac{1}{(\pi)^{N / 2}} \int_{\mathbb{R}^{N}} 1_{\left\{\Psi_{h}(z) \geq 0\right\}} \exp \left(-|z|^{2}\right) d z \leq \frac{1}{2} \\
& \quad-\frac{1}{\sqrt{\pi}}\left(a-\frac{1}{2}\left(\operatorname{Tr}\left(\tilde{A}-\tilde{A}_{1,1}\right)-\frac{n+1}{2} \gamma\right) \frac{\eta}{\beta_{h}}+\circ\left(\frac{\eta}{\beta_{h}}\right) .\right.
\end{aligned}
$$

But the following properties hold true:

$$
\begin{gathered}
\operatorname{Tr}(\tilde{A})=\operatorname{Tr}\left(O\left(D^{2} \phi(x, t) O^{-1}\right)=\operatorname{Tr}\left(D^{2} \phi(x, t)\right)=\Delta \phi(x, t),\right. \\
a=\frac{1}{2} \frac{\partial \phi}{\partial t}(x, t),
\end{gathered}
$$

and if $e=(1, \ldots, 0)$,

$$
\tilde{A}_{1,1}=(\tilde{A} e, e)=\left(D^{2} \phi(x, t) O^{t} e, O^{t} e\right)=\lim _{h} \frac{1}{\beta_{h}^{2}}\left(A_{h} p_{h}, p_{h}\right),
$$

and, recalling that $p_{h} \rightarrow D \phi(x, t)$ and $A_{h} \rightarrow D^{2} \phi(x, t)$,

$$
\tilde{A}_{1,1}=\frac{1}{|D \phi(x, t)|^{2}}\left(D^{2} \phi(x, t) D \phi(x, t), D \phi(x, t)\right) \text {. }
$$

Hence

$$
\begin{aligned}
& \frac{1}{\eta}\left(\frac{1}{2}-\frac{1}{(4 \pi h)^{N / 2}} \int_{\left\{\phi_{h}\left(., t_{h}-h\right) \geq 0\right\}} \exp \left(-\frac{\left|x_{h}-y\right|^{2}}{4 h}\right) d y\right) \\
& \quad \geq \frac{1}{2 \sqrt{\pi}|D \phi(x, t)|}\left(\frac{\partial \phi}{\partial t}-\left(\Delta \phi-\frac{\left(D^{2} \phi D \phi \mid D \phi\right)}{|D \dot{\phi}|^{2}}\right)(x, t)-\frac{n+1}{2} \gamma\right)+\circ(1)
\end{aligned}
$$

for all $\gamma>0$ and the first part of the proposition is proved.

Now we turn to the case when $D \phi(x, t)=0$ and $D^{2} \phi(x, t)=0$. This time $\beta_{h} \rightarrow 0$ and we cannot argue in the same way. Several cases may occur.

1 . Along some subsequence $\beta_{h} \neq 0$ and $\frac{\eta}{\beta_{h}} \rightarrow 0$. In this case, we readily apply the same argument as above. We are led to (14) and easy computations yield

$$
\frac{\beta_{h}}{\eta}\left(\frac{1}{2}-\frac{1}{(4 \pi h)^{N / 2}} \int_{\left\{\phi_{h}\left(. . t_{h}-h\right) \geq 0\right\}} \exp \left(-\frac{\left|x_{h}-y\right|^{2}}{4 h}\right) d y\right) \geq \frac{1}{2 \sqrt{\pi}}\left(\frac{\partial \phi}{\partial t}(x, t)-\frac{n+1}{2} \gamma\right)+o(1)
$$

for any $\gamma>0$ and the conclusion follows easily.

2. Along some subsequence $\beta_{h} \equiv 0$ or $\frac{\eta}{\beta_{h}} \rightarrow+\infty$. Assume by contradiction that $\lim _{h} a_{h}=\frac{1}{2} \frac{\partial \phi}{\partial t}(x, t)>0$. Then the characteristic function of the set

$$
\left\{\beta_{h} z_{1}+\eta\left(\left(A_{h} z, z\right)-a_{h}+O\left(\eta|z|+\eta^{2}\right)\left(|z|^{2}+\eta^{2}\right)\right) \geq 0\right\},
$$

which is the same as the set

$$
\left\{\frac{\beta_{h}}{\eta} z_{1}+\left(\left(A_{h} z, z\right)-a_{h}+O\left(\eta|z|+\eta^{2}\right)\left(|z|^{2}+\eta^{2}\right)\right) \geq 0\right\}
$$


converges almost everywhere to the constant function 0 . Hence

$$
\frac{1}{(4 \pi h)^{N / 2}} \int_{\left\{\phi_{h}\left(., t_{h}-h\right) \geq 0\right\}} \exp \left(-\frac{\left|x_{h}-y\right|^{2}}{4 h}\right) d y \rightarrow 0,
$$

and this is a contradiction since this integral is greater than $\frac{1}{2}$.

3. Along some subsequence $\beta_{h} \neq 0$ and $\frac{\eta}{\beta_{h}} \rightarrow l>0$. Then the characteristic function of the set

$$
\left\{\beta_{h} z_{1}+\eta\left(\left(A_{h} z, z\right)-a_{h}+O\left(\eta|z|+\eta^{2}\right)\left(|z|^{2}+\eta^{2}\right)\right) \geq 0\right\}
$$

which is the same as the set

$$
\left\{z_{1}+\frac{\eta}{\beta_{h}}\left(\left(A_{h} z, z\right)-a_{h}+O\left(\eta|z|+\eta^{2}\right)\left(|z|^{2}+\eta^{2}\right)\right) \geq 0\right\},
$$

converges a.e. to the characteristic function of the set

$$
\left\{z_{1}-\frac{l}{2} \frac{\partial \phi}{\partial t}(x, t) \geq 0\right\}
$$

But we know that

$$
\frac{1}{2}-\frac{1}{(4 \pi h)^{N / 2}} \int_{\left\{\phi_{h}\left(., t_{h}-h\right) \geq 0\right\}} \exp \left(-\frac{\left|x_{h}-y\right|^{2}}{4 h}\right) d y \leq 0,
$$

and by letting $h \rightarrow 0$, we deduce

$$
\frac{1}{2}-\frac{1}{(4 \pi)^{N / 2}} \int_{\left\{z_{1}-\frac{l}{2} \frac{\partial \phi}{\partial t}(x, t) \geq 0\right\}} \exp \left(-|z|^{2}\right) d y \leq 0 .
$$

Consequently

$$
\frac{\partial \phi}{\partial t}(x, t) \leq 0
$$

and the proof is complete.

5. Convergence of the discrete moving front to the continuous one. This section is devoted to the proof of Corollary 1.3. The first step is to prove the following lemma.

LEMMA 5.1. Let $\Omega_{0}$ be a smooth bounded domain in $\mathbb{R}^{N}$. If $\Omega_{0} \subset \bar{B}_{R}$ for some $R>0$ then $\Omega_{n h}^{h} \subset \bar{B}_{R}$ for any $n \in \mathbb{N}$. i.e.,

Proof. We first remark that if we initialize the scheme with a half-space domain,

$$
u_{h}(x, 0)=1_{H}-1_{H^{c}} \quad \text { in } \mathbb{R}^{N},
$$

where $H=\{(p, x)>a\}$ for some $p \in \mathbb{R}^{N}$ and $a \in \mathbb{R}$, then for all $n \in \mathbb{N}$

$$
u_{h}(x, t)=1_{H}-1_{H^{c}} \quad \text { in } \mathbb{R}^{N} .
$$

Therefore we keep at the discrete level the fact that hyperplanes do not move since they have zero mean curvature. 
To prove the lemma, it suffices to use solutions like (15) as supersolutions in the scheme. Indeed since $\Omega_{0} \subset \bar{B}_{R}, \Omega_{0} \subset H_{e}$ for all $|e|=1$ where

$$
H_{e}=\left\{x \in \mathbb{R}^{N} ;(e, x)<R\right\} \text {. }
$$

Therefore

$$
1_{\Omega_{0}}-1_{\Omega_{0}^{c}} \leq 1_{H_{c}}-1_{H_{e}^{c}} \quad \text { in } \mathbb{R}^{N} .
$$

But the scheme is order preserving and therefore we keep this inequality when we iterate the scheme for the two initial data

$$
u_{h}(., n h) \leq 1_{H_{e}}-1_{H_{e}^{c}} \quad \text { in } \mathbb{R}^{N},
$$

for any $n \in \mathbb{N}$. This inequality, which holds true for any $|e|=1$, yields $\Omega_{n h}^{h} \subset \bar{B}_{R}$.

Now we turn to the proof of Corollary 1.3. From the results of Evans and Spruck [10], we know that, since $\Omega_{0}$ is bounded, there is extinction, i.e., $\Gamma_{t}$ collapses in a finite time denoted by $t^{*}$. This information, in addition to Lemma 5.1, will allow us to work on the compact set $K=\bar{B}_{R} \times\left[0, t^{*}+1\right]$.

We first recall that the Hausdorff distance between $F$ and $F^{h}$ is given by

$$
\operatorname{dist}\left(F, F^{h}\right)=\max \left(\max _{(x, t) \in F^{h}} \operatorname{dist}((x, t), F) ; \max _{(x, t) \in F} \operatorname{dist}\left((x, t), F^{h}\right)\right) .
$$

To prove $\operatorname{dist}\left(F, F^{h}\right) \rightarrow 0$, we are going to prove successively that

$$
\max _{(x, t) \in F^{h}} \operatorname{dist}((x, t), F) \rightarrow 0,
$$

and then

$$
\max _{(x, t) \in F} \operatorname{dist}\left((x, t), F^{h}\right) \rightarrow 0 .
$$

To prove the first property, we consider $\varepsilon>0$ and set $K_{\varepsilon}=\{(x, t) \in K$; $\operatorname{dist}((x, t), F) \geq \varepsilon\}$. For any $\varepsilon>0 K_{\varepsilon}$ is a compact subset of $\mathbb{R}^{N} \times(0,+\infty)$ and the main remark is that the function $(x, t) \mapsto \operatorname{sign}(u(x, t))$ is continuous on $K_{\varepsilon}$ since it is locally constant on $K_{\varepsilon}$. It is a classical remark in the theory of viscosity solutions that the equalities

$$
\liminf u_{h}=\limsup u_{h}=\operatorname{sign}(u) \text { on } K_{\varepsilon}
$$

imply the uniform convergence of $u_{h}$ to $\operatorname{sign}(u)$ on $K_{\varepsilon}$. Since these functions assume only the values +1 and -1 , this means that $u_{h}=\operatorname{sign}(u)$ on $K_{\varepsilon}$ for $\mathrm{h}$ small enough. In other words, for $h$ small enough, the discrete front $F^{h}$ is included in the complementary of $K_{\varepsilon}$ in $K$, i.e.,

$$
F^{h} \subset\{(x, t) \in K ; \operatorname{dist}((x, t), F)<\varepsilon\},
$$

and the proof of the first property is complete. Notice that we do not use, in this case, the condition $\bigcup_{t>0} \Gamma_{t} \times\{t\}=\partial\{(x, t) ; u(x, t)>0\}=\partial\{(x, t) ; u(x, t)<0\}$.

We turn to the proof of the second property. The fact that $F$ satisfies the above condition implies that $\operatorname{sign}(u)$ is the unique solution of $(2)$ with initial data $1_{\Omega_{0}}-1_{\Omega_{0}^{c}}$, or equivalently that we have

$$
\left(\operatorname{sign}^{*}(u)\right)_{*}=\operatorname{sign}_{*}(u) \quad \text { in } \mathbb{R}^{N} \times(0,+\infty),
$$


and

$$
\left(\operatorname{sign}_{*}(u)\right)^{*}=\operatorname{sign}^{*}(u) \quad \text { in } \mathbb{I R}^{N} \times(0,+\infty)
$$

In other words, the minimal solution is nothing but the lsc envelope of the maximal one and in the same way the maximal solution is nothing but the usc envelope of the minimal one.

On the other hand, we know that, for any subsequence $h^{\prime}$ going to 0 , the functions $\bar{u}$ and $\underline{u}$, respectively, defined by

$$
\bar{u}(x, t)=\limsup _{\substack{y \rightarrow x \\ n h^{\prime} \rightarrow t}} u_{h^{\prime}}\left(y, n h^{\prime}\right)
$$

and

$$
\underline{u}(x, t)=\liminf _{\substack{y \rightarrow x \\ n h^{\prime} \rightarrow t}} u_{h^{\prime}}\left(y, n h^{\prime}\right)
$$

are viscosity sub- and supersolutions, respectively, of (2) with initial data $1_{\Omega_{0}}-1_{\Omega_{0}^{c}}$. Recalling that $\operatorname{sign}^{*}(u)$ and $\operatorname{sign}_{*}(u)$ are the maximal subsolution and the minimal supersolution, respectively, we get the inequalities

$$
\operatorname{sign}_{*}(u(x, t)) \leq \underline{u}(x, t) \leq \bar{u}(x, t) \leq \operatorname{sign}^{*}(u(x, t)) \quad \text { in } \mathbb{R}^{N} \times(0,+\infty) .
$$

Applying successively the lsc envelope and the usc envelope to (18) and using (16) and (17), we finally conclude that

$$
\liminf _{\substack{y \rightarrow x \\ n h^{\prime} \rightarrow t}} u_{h^{\prime}}\left(y, n h^{\prime}\right)=\operatorname{sign}_{*}(u(x, t)),
$$

and

$$
\limsup _{\substack{y \rightarrow x \\ n h^{\prime} \rightarrow t}} u_{h^{\prime}}\left(y, n h^{\prime}\right)=\operatorname{sign}^{*}(u(x, t)) .
$$

Now assume by contradiction that

$$
\forall h>0, \quad \max _{(x, t) \in F} \operatorname{dist}\left((x, t), F^{h}\right) \geq r>0 .
$$

Since $\mathrm{F}$ is a compact subset of $\mathbb{R}^{N} \times(0,+\infty)$, there is a sequence denoted by $h^{\prime}$ going to 0 and points $\left(x_{h^{\prime}}, t_{h^{\prime}}\right) \in F$ s.t.

$$
\operatorname{dist}\left(\left(x_{h^{\prime}}, t_{h^{\prime}}\right), F^{h^{\prime}}\right) \geq r>0 .
$$

Extracting subsequences if necessary, we may first assume that $\left(x_{h^{\prime}}, t_{h^{\prime}}\right)$ converges to some point $(x, t) \in F$. Recall that $F$ is a closed subset since it is exactly the set $\{u=0\}$. It is then clear that, again up to the extraction of a subsequence, we have one of the following cases: either

$$
\text { for any } h^{\prime}, u_{h}\left(y, n h^{\prime}\right) \equiv 1 \quad \text { if }\left|y-x_{h^{\prime}}\right|+\left|n h^{\prime}-t_{h^{\prime}}\right|<r \text {, }
$$


or

$$
\text { for any } h^{\prime}, u_{h}\left(y, n h^{\prime}\right) \equiv-1 \quad \text { if }\left|y-x_{h^{\prime}}\right|+\left|n h^{\prime}-t_{h^{\prime}}\right|<r .
$$

Consider for example the first case (the other one is treated similarly). It implies

$$
\liminf _{\substack{y \rightarrow x \\ n h^{\prime} \rightarrow t}} u_{h^{\prime}}\left(y, n h^{\prime}\right)=1=\operatorname{sign}_{*}(u(x, t)) .
$$

This provides a contradiction since $(x, t) \in F$ and $\operatorname{sign}_{*}(u(x, t))=-1$ on $F$. The proof is complete.

Acknowledgments. The authors thank L. C. Evans for sending us a preliminary version of his work and S. J. Osher for sending several related articles, in particular [12]. They also thank P. E. Souganidis for pointing out an error in a previous version of this paper. They are grateful to the referee whose relevant remarks lead to significant improvements in the draft of this paper. They thank Hervé Choplin for his help in the final typesetting of this paper.

\section{REFERENCES}

[1] G. Barles and B. Perthame, Discontinuous solutions of deterministic optimal stopping problems, Math. Modelling Numer. Anal., 21 (1987) pp. 557-579.

[2] G. Barles, H. M. Soner, AND P. E. Sovganidis, Fronts Propagations and Phase Fields Theory, SIAM J. Control Optim., 31 (1993), pp. 439-469.

[3] G. Barles AND P. E. Souganidis, Convergence of approximation schemes for fully non-linear equations, Asymptotic Anal., 4 (1989), pp. 271-283.

[4] J. Bence, B. Merriman, AND S. Osher, Diffusion generated motion by mean curvature, preprint.

[5] Y.-G. Chen, Y. Giga, AND S. Goto, Uniqueness and existence of viscosity solutions of generalized mean curvature flow equations, J. Differential Geom., 33 (1991), pp. 749-786.

[6] M. Crandall, L. C. Evans, and P. L. Lions, Some properties of viscosity solutions of Hamilton-Jacobi Equations, Trans. Amer. Math. Soc., 282 (1984), pp. 487-502.

[7] M. Crandall, H. IshiI, AND P. L. Lions, User's guide to viscosity solutions of second order Partial differential equations, Bull. Amer. Soc., 27 (1992), pp. 1-67.

[8] M. CRAndall and P. L. Lions, Viscosity solutions of Hamilton-Jacobi Equations, Trans. Amer. Math. Soc., 277 (1983), pp. 1-42.

[9] L. C. Evans, Convergence of an algorithm for mean curvature motion, preprint.

[10] L. C. Evans AND J. Spruck, Motion of level sets by mean curvature, I, J. Differential Geom., 33 (1991), pp. 635-681; II, Trans. Amer. Math. Soc., 330 (1992), pp. 321-332; III, J. Geom. Anal., 2 (1992), pp. 121-150; IV, J. Geom. Anal., to appear.

[11] H. IsHII, Hamilton-Jacobi Equations with discontinuous Hamiltonians on arbitrary open sets, Bull. Fac. Sci. Engrg. Chuo Univ. Ser. I Math., 26 (1985), pp. 5-24.

[12] P. MASCARENHAS, Diffusion generated motion by mean curvature, Cam report, Math Dept., University of California, Los Angeles, 1992.

[13] S. Osher AND J. A. Sethian, Fronts moving with curvature-dependent speed: Algorithms based on Hamilton-Jacobi equations. J. Comput. Phys., 79 (1988), pp. 12-49.

[14] H. M. SONER, Motion of a set by the mean curvature of its boundary. J. Differential Equations, 101 (1993) pp. 313-372. 